CLINICAL STUDY

\title{
Study of carbohydrate metabolism indices and adipocytokine profile and their relationship with androgens in polycystic ovary syndrome after menopause
}

\author{
Marios C Markopoulos ${ }^{1}$, George Valsamakis ${ }^{2}$, Evangelia Kouskouni ${ }^{3}$, Anastassios Boutsiadis ${ }^{2}$, \\ Ioannis Papassotiriou ${ }^{4}$, George Creatsas ${ }^{1}$ and George Mastorakos ${ }^{1,2}$ \\ ${ }^{1}$ Second Department of Obstetrics and Gynecology, ${ }^{2}$ Endocrine Unit and ${ }^{3}$ Department of Clinical Biochemistry, Athens University Medical School, \\ Aretaieion University Hospital, 10682 Athens, Greece and ${ }^{4}$ Department of Clinical Biochemistry, Aghia Sophia Children's Hospital, Athens, Greece \\ (Correspondence should be addressed to G Mastorakos who is now at 3, Neofytou Vamva Street, Athens 10674, Greece; Email: \\ mastorakg@ath.forthnet.gr)
}

\begin{abstract}
Objective: Hyperandrogenism, insulin resistance, and altered adipocytokine levels characterize polycystic ovary syndrome (PCOS) women of reproductive age. Hyperandrogenism persists in postmenopausal PCOS women. In the latter, this study aimed at investigating carbohydrate metabolism, adipocytokines, androgens, and their relationships.

Subjects and methods: Blood sampling from overweight postmenopausal women (25 PCOS and 24 ageand BMI-matched controls) at baseline and during oral glucose tolerance test for measurement of insulin and glucose levels, baseline leptin, adiponectin, visfatin, retinol-binding protein 4, lipocalin-2, androgen, and high-sensitivity C-reactive protein (hs-CRP) levels and for calculation of insulin sensitivity (glucose-to-insulin ratio (G/I), quantitative insulin sensitivity check index, and insulin sensitivity index (ISI)), resistance (homeostasis mathematical model assessment-insulin resistance (HOMA-IR)), secretion ( $\Delta$ of the area under the curve of insulin ( $\triangle$ AUCI), first-phase insulin secretion (1st PHIS), and second-phase insulin secretion (2nd PHIS)), and free androgen indices (FAI).

Results: PCOS women had higher insulin secretion indices, hs-CRP, androgen, and FAI levels than controls without differing in baseline glucose, insulin and adipocytokines levels, insulin sensitivity, and resistance indices. In PCOS women, FAI levels correlated positively with baseline insulin, $\triangle A U C I$, HOMA-IR, and $\triangle$ AUCG and negatively with G/I; hs-CRP levels correlated positively with $\triangle$ AUCI and negatively with ISI. PCOS status, waist circumference, and 17-hydroxyprogesterone (17-OHP) levels were positive predictors for $\triangle$ AUCI. In all women, waist circumference was a negative predictor for ISI; 17-OHP and FAI levels were positive predictors respectively for baseline insulin levels and for 1st PHIS and 2nd PHIS.

Conclusions: Early postmenopausal PCOS women are characterized by hyperinsulinemia but attenuated insulin resistance. PCOS status and waist circumference are predictors of hyperinsulinemia while insulin sensitivity correlates negatively with FAI. The differences reported in adipocytokine levels between PCOS and non-PCOS women in reproductive years seem to disappear after menopause.
\end{abstract}

European Journal of Endocrinology 168 83-90

\section{Introduction}

Polycystic ovary syndrome (PCOS) is the most common endocrinopathy in the female population (1) and is characterized by both reproductive and metabolic aberrations. Insulin resistance and compensatory hyperinsulinemia are present in about $50-70 \%$ of premenopausal PCOS women (2). Consequently, the latter present a three- to seven-fold higher risk of developing impaired glucose intolerance and type 2 diabetes mellitus (T2DM) compared with the general population (3).

Insulin resistance in PCOS is considered to be the result of both intrinsic and acquired functional disturbances. The positive correlation between insulin resistance and hyperandrogenism in PCOS women of reproductive age is well documented. Androgens reduce insulin sensitivity in skeletal muscles (4), while increased testosterone levels induce insulin resistance in the adipocytes of PCOS women in vitro (5). In addition, insulin resistance via the resulting hyperinsulinemia is associated with enhanced ovarian androgen production in women of reproductive age with PCOS (6). Conversely, others report the presence of insulin resistance in PCOS only in the face of elevated androgen levels (7).

Abdominal obesity is a frequent characteristic in patients with PCOS (8). Excessive visceral adipose tissue 
accumulation induces insulin resistance through various mechanisms including adipocytokine production. In fact, adipocytokine secretion is dysregulated in PCOS women of reproductive age, while altered adipocytokine levels, including leptin, adiponectin, and more recently visfatin, retinol-binding protein 4 (RBP4), and lipocalin-2, have been implicated in aberrations of carbohydrate metabolism and in the pathophysiology of insulin resistance $(9,10,11)$. Moreover, there seems to be a complex association between insulin resistance, obesity, and low-grade chronic inflammation in PCOS. High-sensitivity C-reactive protein (hs-CRP), a stable marker of chronic subclinical inflammation, has been inversely correlated with insulin sensitivity in women with and without PCOS $(12,13)$ and has been suggested as an independent predictor of the risk for T2DM (14).

The evolution of the metabolic profile of PCOS women into menopause is not well explored as yet. Long-term follow-up studies of women with PCOS show significantly higher prevalence of glucose metabolism disturbances than in healthy women, indicating that the risk for T2DM is sustained as they reach menopause (15). There is evidence that hyperandrogenism resolves partially in women with PCOS in perimenopause (16), although, as we have recently shown, androgens of both ovarian and adrenal origin are higher in postmenopausal women with PCOS compared with controls (17). These changes might influence insulin resistance in PCOS women. In addition, data regarding adipocytokines and CRP levels in postmenopausal PCOS women are scarce.

Thus, the aim of this study was to investigate carbohydrate metabolism indices and adipocytokine levels as markers of adipose tissue as well as their correlations with the androgen profile in PCOS women at early postmenopause compared with non-PCOS women. To do so, we measured insulin secretion and resistance indices during a $75 \mathrm{~g}$ oral glucose tolerance test (OGTT), as well as baseline androgen (17-hydroxyprogesterone (17-OHP), $\Delta_{4}$-androstenedione $\left(\Delta_{4} \mathrm{~A}\right)$, DHEAS, total testosterone, sex hormone-binding globulin (SHBG), free androgen index (FAI), and estradiolto-total testosterone ratio), fasting adipocytokine (leptin, adiponectin, visfatin, RBP4, and lipocalin-2), and hs-CRP levels, and investigated their correlations in postmenopausal PCOS and control women.

\section{Materials and methods}

\section{Patients and control subjects}

Twenty-five postmenopausal women with PCOS and 24 age- and BMI-matched healthy controls were included in this study (Table 1). All women were followed in our unit before and after their pregnancies until after their menopause. Thus, each subject had a detailed record of their menses and of their clinical and biochemical status of hyperandrogenism. Diagnosis of the menopausal status was based on a history of more than 12 months of amenorrhea after the final menstrual period and on serum FSH levels > $30 \mathrm{IU} / \mathrm{l}$. Diagnosis of PCOS was confirmed retrospectively and was based on the National Institute of Health criteria established in 1990 (18). All subjects with a history of oligomenorrhea (less than eight menses per year) and signs of clinical and/or biochemical hyperandrogenism in their record were diagnosed as patients with PCOS. The retrospective diagnosis of the PCOS status in the women participating in this study, although based on their detailed medical records, could be considered as a limitation of the study. Up to now, there are no defined criteria for the de novo diagnosis of PCOS in menopause. All control subjects were characterized by regular menses and by absence of clinical and biochemical

Table 1 Anthropometric characteristics, baseline carbohydrate metabolism parameters, and OGTTderived indices (mean \pm S.E.M.) in postmenopausal PCOS and control women.

\begin{tabular}{lccc}
\hline & PCOS $(n=25)$ & Controls $(n=24)$ & $\boldsymbol{P}$ \\
\hline Age (years) & $55.37 \pm 4.84$ & $58.45 \pm 6.59$ & $\mathrm{NS}$ \\
Years since menopause & $4.57 \pm 2.81$ & $5.45 \pm 3.72$ & $\mathrm{NS}$ \\
Weight (kg) & $73.1 \pm 5.42$ & $65.18 \pm 9.86$ & $\mathrm{NS}$ \\
BMI & $27.4 \pm 2.5$ & $26.03 \pm 2.87$ & $\mathrm{NS}$ \\
Waist circumference (cm) & $98.3 \pm 9.39$ & $88.70 \pm 8.99$ & $<0.05$ \\
Waist-to-hip ratio & $0.90 \pm 0.10$ & $0.84 \pm 0.06$ & $<0.05$ \\
Total body fat \% & $42.81 \pm 4.22$ & $40.38 \pm 4.89$ & $\mathrm{NS}$ \\
Fasting glucose (mg/dl) & $93.2 \pm 13.25$ & $92 \pm 15.64$ & $\mathrm{NS}$ \\
Fasting insulin $(\mu \mathrm{IU} / \mathrm{ml})$ & $8.36 \pm 4.1$ & $6.04 \pm 3.46$ & $\mathrm{NS}$ \\
G/I & $14.79 \pm 9.24$ & $17.87 \pm 8.68$ & $\mathrm{NS}$ \\
HOMA-IR & $2.05 \pm 1.25$ & $1.61 \pm 0.86$ & $\mathrm{NS}$ \\
QUICKI & $0.35 \pm 0.04$ & $0.37 \pm 0.03$ & $\mathrm{NS}$ \\
ISI & $0.12 \pm 0.005$ & $0.13 \pm 0.002$ & $\mathrm{NS}$ \\
1st PHIS & $905.13 \pm 129.68$ & $277.90 \pm 20.84$ & $<0.01$ \\
2nd PHIS & $433.04 \pm 55.82$ & $230.76 \pm 12.50$ & $<0.01$ \\
$\Delta$ AUCG & $4705.36 \pm 442.31$ & $4605.25 \pm 514.23$ & $\mathrm{NS}$ \\
$\Delta$ AUCl & $9113.21 \pm 1215.67$ & $6612.94 \pm 864.87$ & $<0.05$ \\
\hline
\end{tabular}

NS, nonsignificance. 
signs of hyperandrogenism during their reproductive years. Women with PCOS-like states such as hyperprolactinemia, nonclassical adrenal hyperplasia, Cushing's syndrome, and ovarian or adrenal androgen-secreting tumors were excluded. Exclusion criteria also comprised a history of oophorectomy or hysterectomy, T2DM, and use of any medication known to affect metabolic parameters and/or hormonal indices, including statins, metformin, or antiobesity drugs, during or before the study. Among all subjects included in the study, three PCOS women and four control women were smokers $(<10$ cigarettes/day) at the time of the study. The study was approved by our institution ethics committee and all subjects gave written informed consent.

\section{Protocol}

A complete medical history was obtained from all subjects. All subjects underwent standard anthropometric examination including weight, height, and waist and hip circumference measurements. Skinfold thickness at four body sites (biceps, triceps, suprailiac, and subscapular skinfold) was also measured. Blood sampling was obtained at $0800 \mathrm{~h}$ from all subjects, after a 12-h fast, for measurements of glucose, insulin, leptin, adiponectin, visfatin, RBP4, lipocalin-2, FSH, $\mathrm{LH}$, estradiol, prolactin, progesterone, 17-OHP, $\Delta_{4} \mathrm{~A}$, DHEAS, total testosterone, SHBG, and hs-CRP levels at baseline followed by a 2-h OGTT.

\section{Methods}

Anthropometry Weight in kilograms and height in meters were measured using an electronic scale and a stadiometer respectively, and BMI in kilograms per square meter was calculated (Table 1). Maximum hip and waist circumferences in centimeters were measured in duplicate using a flexible tape and waist-to-hip ratio was calculated. Skinfold thickness was measured on the right side of the body using a Harpenden skinfold caliper (Assist Creative Resources Ltd., Wrexham, UK) in triplicate to the nearest $0.1 \mathrm{~mm}$. Biceps and triceps skinfold thicknesses were measured at the midpoint of the upper arm, between the acromion process, and the tip of the bent elbow. Suprailiac skinfold was pinched at $2-3 \mathrm{~cm}$ above the iliac crest on the lateral side and midaxillary line. Subscapular thickness was measured at the natural fold $\sim 2-3 \mathrm{~cm}$ below the shoulder blade at an oblique angle. Percentage of total body fat was estimated from skinfold thicknesses (19).

Oral glucose tolerance test At $0800 \mathrm{~h}$, patients, after a 12-h fast, were placed on bed rest for the duration of the procedure and were given $75 \mathrm{~g}$ glucose orally. Blood sampling was performed at 5, 15, 30, 60, 90, and $120 \mathrm{~min}$ after glucose administration for the measurement of glucose and insulin levels.
Hormone and adipocytokine assays Plasma and serum from all blood samples were separated in a centrifuge within $30 \mathrm{~min}$ from blood withdrawal, stored in polystyrene tubes, and frozen at $-70{ }^{\circ} \mathrm{C}$ until assayed. Serum insulin levels were measured by a chemiluminescent microparticle immunoassay (CMIA; Abbott Laboratories, Abbott Park, IL, USA); detection limit (DL) was $1.0 \mu \mathrm{U} / \mathrm{ml}$; and total coefficient of variation $(\mathrm{CV})$ was $<7 \%$. Serum DHEAS and SHBG levels were measured by an electrochemiluminescence immunoassay (ECLIA; Roche Diagnostics, Mannheim, Germany); DL was $0.1 \mu \mathrm{g} / \mathrm{dl}$ and $0.35 \mathrm{nmol} / \mathrm{l}$ respectively; total $\mathrm{CV}$ was $<4.7$ and $<5.6 \%$ respectively. Serum total testosterone, FSH, LH, estradiol, progesterone, and prolactin levels were measured by a microparticle enzyme immunoassay (MEIA; Abbott Laboratories); DL was $0.1 \mathrm{ng} / \mathrm{ml}, 0.4 \mathrm{mIU} / \mathrm{ml}$, $0.5 \mathrm{mIU} / \mathrm{ml}, 20 \mathrm{pg} / \mathrm{ml}, 0.2 \mathrm{pg} / \mathrm{ml}$, and $0.6 \mathrm{ng} / \mathrm{ml}$ respectively; total $\mathrm{CV}$ was found to be $<12,5.1-10.1$, $5.2-10,4.4-13.1,3.4-11.7$, and $3.4-6.3 \%$ respectively. FAI was calculated as the ratio of total testosterone $(\mathrm{nmol} / \mathrm{l})$ to SHBG (nmol/l) levels $\times 100$ (20). Estradiolto-total testosterone ratio was calculated, as a measure of relative hyperandrogenism, after conversion of their values to the same units $(\mathrm{pg} / \mathrm{ml})$. Estrone, although being the principal estrogen in women after menopause, was not measured because the appropriate methodology for the measurement of estrone levels is quantitative high-performance liquid chromatography-tandem mass spectrometry to which we had no access (21). Serum $\Delta_{4} \mathrm{~A}, 17-\mathrm{OHP}$, and adiponectin levels were measured by ELISA (IBL-America, Minneapolis, MN, USA; BioSource, Nivelles, Belgium; and Orgenium, Vantaa, Finland respectively); DL was 2, 0.03, and $0.185 \mathrm{ng} / \mathrm{ml}$ respectively; total $\mathrm{CV}$ was $<12,<8$, and $<12 \%$ respectively. Serum leptin levels were measured by a fluorescent immunoassay with Luminex xMAP technology (Millipore Corp., Billerica, MA, USA); DL was $35 \mathrm{pg} / \mathrm{ml}$; interassay $\mathrm{CV}$ was $7 \%$. Serum visfatin levels were measured using a visfatin C-terminal ELISA kit (Phoenix Pharmaceuticals, Inc., Belmont, CA, USA); DL was $1.85 \mathrm{ng} / \mathrm{ml}$. Serum lipocalin-2 and RBP4 levels were measured by a solid-phase ELISA method (R\&D Systems, Minneapolis, MN, USA); DL was 0.012 and $0.053 \mathrm{ng} / \mathrm{ml}$ respectively; interassay $\mathrm{CV}$ was 5.6-7.9 and $5.8-8.6 \%$ respectively. Because some adipocytokines are associated with central adiposity, we calculated the adipocytokine-to-waist circumference ratio to better reflect this association.

Glucose and hs-CRP assays Hexokinase/G-6-PDH methodology was used for glucose measurements (Abbott Laboratories); DL was $2.5 \mathrm{mg} / \mathrm{dl}$; total CV was $<5 \%$. Serum hs-CRP levels were measured with a fully automated latex particle-enhanced immunonephelometric assay (Dade Behring, Siemens Healthcare Diagnostics, North Salt Lake, UT, USA); DL was $0.175 \mathrm{mg} /$; interassay $\mathrm{CV}$ was $<7 \%$. 
Indices of carbohydrate metabolism The following mathematical calculations were performed from fasting and OGTT-derived measurements in order to estimate insulin sensitivity and resistance, $\beta$-cell function, and insulin secretion indices:

Carbohydrate metabolism indices derived from fasting values Insulin sensitivity indices:

Glucose-to-insulin ratio $(\mathrm{G} / \mathrm{I})=$ glucose at baseline $(\mathrm{mg} / \mathrm{dl}) /$ insulin at baseline $(\mathrm{mmol} / \mathrm{l})(22)$.

Quantitative insulin sensitivity check index (QUICKI) $=1 /(\log ($ insulin $)(\mu \mathrm{IU} / \mathrm{ml})+\log ($ glucose $)(\mathrm{mg} / \mathrm{dl}))(23)$.

Insulin resistance index:

Homeostasis mathematical model assessment-insulin resistance $($ HOMA-IR $)=($ insulin at baseline $(\mathrm{pmol} / \mathrm{l}) \times$ glucose at baseline $(\mathrm{mmol} / \mathrm{l}))(24)$.

Carbohydrate metabolism indices derived from OGTT results To avoid inaccurate estimation of insulin sensitivity by fasting insulin, QUICKI, and G/I as it has been suggested in the past (25), we also employed the insulin sensitivity index (ISI) (26).

$\mathrm{ISI}=0.226-(0.0032 \times \mathrm{BMI})-(0.0000645 \times \mathrm{insu}-$ lin at $120 \mathrm{~min}(\mathrm{pmol} / \mathrm{l}))-(0.00375 \times$ glucose at $90 \mathrm{~min}(\mathrm{mmol} / \mathrm{l}))(26)$.

$\beta$-Cell insulin secretion indices:

Predicted index of the first phase of insulin secretion $(1$ st $\mathrm{PHIS})=1283+(1.289 \times$ insulin at $30 \mathrm{~min}$ $(\mathrm{pmol} / \mathrm{l}))-(138.7 \times$ glucose at $30 \mathrm{~min}(\mathrm{mmol} / \mathrm{l}))+$ $(3.772 \times$ insulin at baseline $(\mathrm{pmol} / \mathrm{l}))$ and predicted index of the second phase of insulin secretion (2nd PHIS $)=287+(0.4164 \times$ insulin at $30 \mathrm{~min}(\mathrm{pmol} / \mathrm{l}))-$ $(26.07 \times$ glucose at $30 \mathrm{~min}(\mathrm{mmol} / \mathrm{l}))+(0.9226 \times$ insulin at baseline (pmol/l)) (26).

OGTT-derived insulinemia and glycemia indices:

The $\Delta$ of the area under the curve (AUC) of insulin $(\triangle \mathrm{AUCI})$ and glucose $(\triangle \mathrm{AUCG})$ was calculated after subtraction of insulin and glucose value respectively at $0 \mathrm{~min}$ from each of the 5-, 15-, 30-, 60-, 90-, and 120-min insulin and glucose values respectively by application of the trapezoidal rule on the area of calculation.

\section{Statistical analysis}

Data with a Gaussian distribution are expressed by mean \pm s.E.M. and data with a skewed distribution are expressed as median and interquartile range. Hormonal and clinical parameters were compared with Student's $t$-test for normally distributed variables and with the Mann-Whitney $U$ test for variables with a skewed distribution. Glucose and insulin levels during OGTT were normally distributed and were compared between postmenopausal women with PCOS and controls by one-way ANOVA for repeated measures. Multiple regression analysis was performed to investigate correlations among each carbohydrate metabolism parameter to hs-CRP and to each androgen and adipocytokine using the PCOS/control status as covariate in the whole group of the women studied. By this model, the interaction of the status with the independent variable was taken into account in each analysis. To define predictive variables (predictors), backward stepwise multiple regression analysis was performed. Carbohydrate metabolism parameters were employed as dependent variables while waist circumference, FAI, 17-OHP and hs-CRP levels, and the PCOS/control status as independent variables. $P$ value $<0.05$ was considered statistically significant. All statistical analyses were performed using Statistica 6.0 software (StatSoft, Inc., Tulsa, USA).

\section{Results}

\section{Anthropometric characteristics, baseline carbohydrate metabolism parameters, and OGTT-derived indices}

Waist circumference and waist-to-hip ratio values were higher $(P<0.05)$ in postmenopausal PCOS women compared with controls (Table 1). Age, number of years since menopause, weight, BMI, percentage of total body fat, fasting glucose and insulin levels, baseline and OGTT-derived insulin resistance and sensitivity indices did not differ significantly between postmenopausal PCOS and control women. Glucose and insulin levels during OGTT are presented in Fig. 1 (A and B respectively). During OGTT, postmenopausal PCOS women had higher $\triangle$ AUCI $(P<0.05)$, 1st PHIS $(P<0.01)$, and 2 nd PHIS $(P<0.01)$ values than control women.

\section{Baseline hormones, adipocytokines, and hs-CRP levels}

Postmenopausal PCOS women had higher baseline progesterone $(P<0.05), 17-\mathrm{OHP}(P<0.05), \Delta_{4} \mathrm{~A}$ $(P<0.05)$, DHEAS $(P<0.05)$, total testosterone $(P<0.05)$ and FAI $(P<0.001)$ levels, and lower SHBG $(P<0.01)$ and estradiol-to-total testosterone ratio $(P<0.01)$ levels than control women. $\mathrm{LH}, \mathrm{FSH}$, estradiol, and prolactin levels did not differ between postmenopausal PCOS and control women, while postmenopausal PCOS women had higher hs-CRP levels $(P<0.01)$ compared with controls (Table 2$)$.

There was no significant difference in fasting leptin, adiponectin, visfatin, and RBP4 levels between the two groups, while PCOS women presented a trend of higher lipocalin-2 levels than control women $(P=0.07)$. All ratios of each adipocytokine levels to waist circumference did not differ between the two groups, except that of lipocalin-2 levels to waist circumference, which was higher $(P<0.05)$ in postmenopausal PCOS than in control women. 

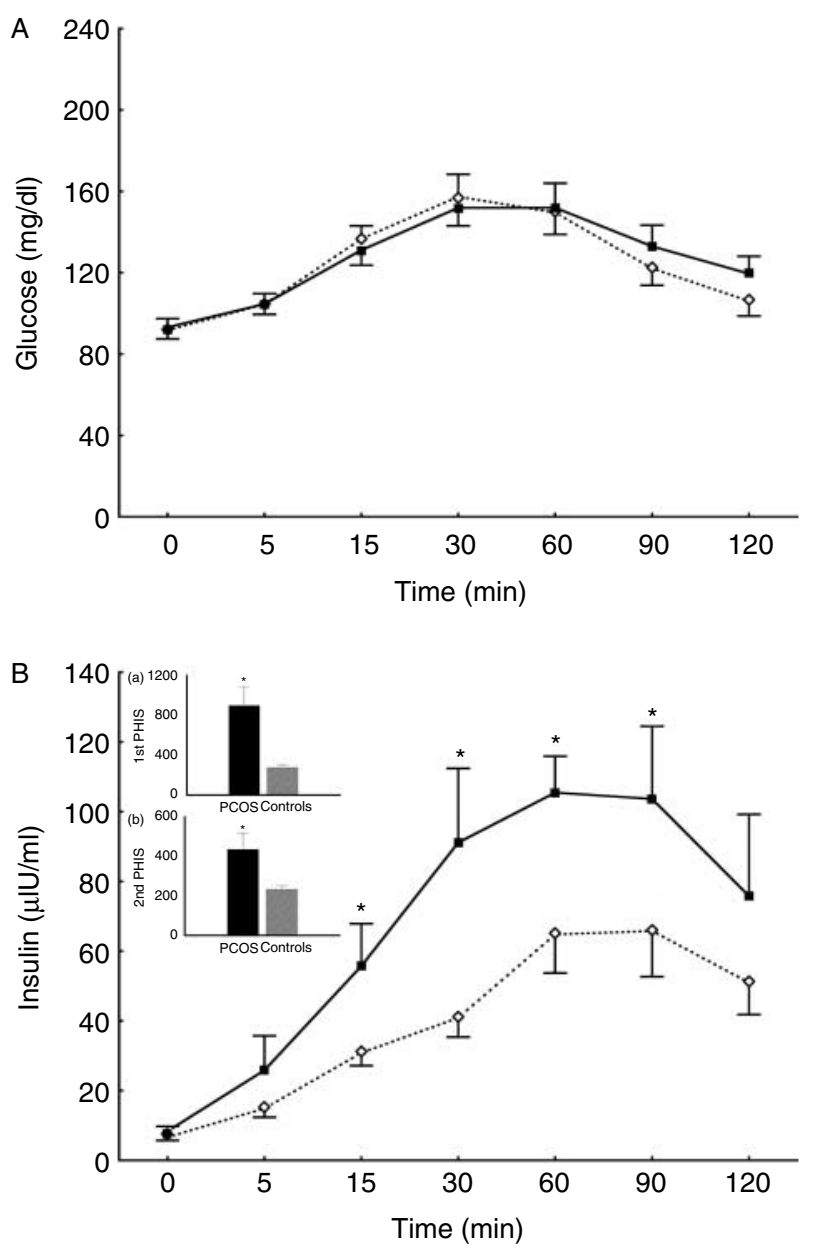

Figure 1 Glucose (A) and insulin (B) levels (mean \pm s.E.M.) during OGTT in postmenopausal PCOS (straight line) and control (dotted line) women. There was no difference in glucose levels at each time-point between postmenopausal PCOS and control women, while insulin levels at 15, 30, 60, and 90 min after glucose administration were higher in postmenopausal PCOS than in control women (one-factor ANOVA repeated measures). Inset (a) and (b) present the predicted index of first-phase insulin secretion (1st PHIS) and second-phase insulin secretion (2nd PHIS) levels (mean \pm S.E.M.) respectively in postmenopausal PCOS (black columns) and control (lineated columns) women during OGTT. Asterisk $\left(^{*}\right)$ indicates a statistically significant difference $(P<0.05)$ from controls.

\section{Correlations}

By employing multiple regression analysis with the $\mathrm{PCOS} /$ control status included as covariate, significant correlations among carbohydrate metabolism parameters and androgen, adipocytokine, or hs-CRP levels emerged with regard to the PCOS group (Table 3).

\section{Predictors of carbohydrate metabolism parameters}

According to backward stepwise multiple regression analysis PCOS status, waist circumference and 17-OHP levels were significant positive predictors for $\triangle \mathrm{AUCI}$ and total AUC of insulin (TAUCI). Waist circumference was a significant negative predictor for ISI while 17-OHP and FAI levels were significant positive predictors for baseline insulin levels and 1st PHIS and 2nd PHIS respectively.

\section{Discussion}

We found that postmenopausal PCOS women presented with hyperinsulinemia during OGTT compared with age- and BMI-matched controls. Glucose levels as well as insulin sensitivity and resistance indices at baseline and during OGTT did not differ between the two groups. Insulin resistance reports on postmenopausal PCOS women are scarce. In postmenopausal PCOS women, Puurunen et al. (27) found hyperinsulinemia during OGTT as well, while baseline insulin and glucose levels, ISI, Matsuda and HOMA-IR indices, and OGTT glucose levels did not differ between the PCOS and the control groups. Thus, it seems that in early postmenopause, insulin resistance does not differ between PCOS and control women, in contrast to the well-documented higher insulin resistance between them during reproductive age (28). Insulin secretion, however, is higher in early postmenopausal PCOS than in control women, indicating that $\beta$-cell function maintains its enhanced response in the former. This hyperinsulinemia in PCOS during early postmenopause could be attributed to $\beta$-cell hypertrophy persisting from its adaptation to the increased insulin resistance of reproductive years.

We have also found that postmenopausal PCOS women presented with higher androgen levels of both adrenal and ovarian origin compared with controls, in accordance with our recent report (17). In postmenopausal PCOS women, FAI levels correlated positively with fasting and OGTT insulin levels and with OGTT glucose levels. They also correlated positively and negatively with insulin resistance and sensitivity indices respectively. In addition, when all women were taken into account, they were the best positive predictors for insulin secretion indices (1st PHIS and 2nd PHIS) while 17-OHP levels were the best positive predictors for fasting insulin levels. In PCOS women of reproductive age, insulin resistance leads via hyperinsulinemia to enhanced ovarian androgen production (6), while elevated androgen levels have an unfavorable impact on insulin sensitivity (29). Thus, it seems that, in PCOS women in early postmenopause, the positive correlation between androgen levels and insulin resistance persists. During menopause, insulin resistance worsens in nonPCOS women $(30,31)$, while PCOS women over 35 years up to early menopause present with lower sensitivity but a well-preserved $\beta$-cell function in comparison with controls (15). Furthermore, total and free testosterone levels decrease in women with PCOS before menopause compared with their early reproductive age, remaining, however, higher than those of 
Table 2 Baseline hormones (median (25th to 75th quartile)), adipocytokine, and hs-CRP levels (mean \pm S.E.M.) in postmenopausal PCOS and control women.

\begin{tabular}{llll}
\hline & PCOS $(n=25)$ & Controls $(n=24)$ & $P$ \\
\hline FSH $(\mathrm{mlU} / \mathrm{ml})$ & $73.4(46.4-90.1)$ & $75.5(40.8-91)$ & $\mathrm{NS}$ \\
LH $(\mathrm{mlU} / \mathrm{ml})$ & $36.1(26.4-42.1)$ & $25.8(19.2-40.4)$ & $\mathrm{NS}$ \\
Estradiol $(\mathrm{pg} / \mathrm{ml})$ & $25.4(21.8-29.2)$ & $29.6(26.7-39.9)$ & $\mathrm{NS}$ \\
Prolactin $(\mathrm{ng} / \mathrm{ml})$ & $8.1(7.1-12.6)$ & $7.3(5-9.6)$ & $<0.05$ \\
Progesterone $(\mathrm{ng} / \mathrm{ml})$ & $0.3(0.3-0.4)$ & $0.2(0.1-0.2)$ & $<0.05$ \\
$17-O H P(\mathrm{ng} / \mathrm{ml})$ & $0.53(0.48-0.58)$ & $0.34(0.26-0.42)$ & $<0.05$ \\
$\Delta_{4}$ A $(\mathrm{ng} / \mathrm{dl})$ & $220(151-358)$ & $111(80-162)$ & $<0.05$ \\
DHEAS $(\mathrm{ng} / \mathrm{ml})$ & $1495(601-1597)$ & $644(396-964)$ & $<0.05$ \\
Total testosterone $(\mathrm{ng} / \mathrm{ml})$ & $0.49(0.39-0.61)$ & $0.35(0.21-0.4)$ & $<0.01$ \\
SHBG $(\mathrm{nmol} / \mathrm{l})$ & $44(25-52.6)$ & $70(55.4-99.2)$ & $<0.001$ \\
FAl & $3.86(3.3-5.3)$ & $1.46(1.12-1.7)$ & $<0.01$ \\
Estradiol/total testosterone & $63.02(35.30-70.27)$ & $114.81(99-280.95)$ & $\mathrm{NS}$ \\
Leptin $(\mathrm{pg} / \mathrm{ml})$ & $17542 \pm 1158$ & $21334 \pm 3542$ & $\mathrm{NS}$ \\
Adiponectin $(\mu \mathrm{g} / \mathrm{ml})$ & $22.54 \pm 3.33$ & $26.87 \pm 3.40$ & $\mathrm{NS}$ \\
Visfatin $(\mathrm{ng} / \mathrm{ml})$ & $15.4 \pm 2.37$ & $12.99 \pm 0.40$ & $\mathrm{NS}$ \\
RBP4 $(\mathrm{mg} / \mathrm{l})$ & $45.22 \pm 4.42$ & $39.76 \pm 2.63$ & $<0.05$ \\
Lipocalin-2 $(\mathrm{ng} / \mathrm{ml})$ & $60.16 \pm 10.84$ & $47.20 \pm 6.46$ & \\
hs-CRP $(\mathrm{mg} / \mathrm{l})$ & $2.97 \pm 1.3$ & $1.06 \pm 0.3$ & \\
\hline
\end{tabular}

NS, nonsignificance.

age-matched control women (16). Interestingly, in this study, during OGTT, $\triangle$ AUCG did not differ between overweight postmenopausal PCOS and control women while $\triangle$ AUCI was higher in the former. This response resembles that observed by Dunaif et al. (28) between lean PCOS and control women of reproductive age. One might suggest that after menopause, insulin sensitivity improves in PCOS women and worsens in control women due to the relative decrease and increase respectively of hyperandrogenism compared with premenopause. This might explain why upon early postmenopause there is no significant difference in insulin resistance between PCOS and control women, while hyperinsulinemia in PCOS women persists.

In this study, hs-CRP levels were higher in postmenopausal PCOS compared with controls, in accordance with the findings of studies in premenopausal PCOS women (32). Apparently, this is due to the higher abdominal fat accumulation of the latter compared with controls as well as with their PCOS status $(32,33)$. In PCOS, hs-CRP levels correlated positively with insulin secretion and negatively with insulin sensitivity indices. CRP levels correlate positively with baseline insulin levels in overweight postmenopausal women with normal and impaired OGTT (34). Interestingly, in this study, hyperinsulinemia was best predicted by PCOS status, 17-OHP levels and waist circumference, among PCOS status, waist circumference, FAI, 17-OHP, and hs-CRP levels as independent variables, indicating that the PCOS status per se increases hyperinsulinemia after menopause. Of note, in the postmenopausal women of this study, waist circumference predicts insulin sensitivity independently of the PCOS status.

In this study, postmenopausal overweight PCOS women had higher abdominal fat accumulation than their age- and BMI-matched controls, without differing in total body fat percentage. PCOS is frequently characterized by abdominal adiposity regardless of BMI (35), while aging and possibly menopause are also associated with fat mass increase and redistribution to the abdominal area (36). Adipocytokine levels represent an important link between adipose tissue and insulin resistance. Although some adipocytokines are found increased in parallel to abdominal adiposity $(37,38)$, in this study, there was no difference in leptin, adiponectin, visfatin, RBP4, and lipocalin-2 levels between PCOS and control women. In premenopausal PCOS women with increased insulin resistance, adiponectin and leptin levels are respectively lower and similar to their levels in non-PCOS women after adjustment for obesity (39). Most studies report higher levels of visfatin, an insulinomimetic hormone, in

Table 3 By employing multiple regression analysis, in which the PCOS/control status was included as a covariate, significant $(P<0.05)$ correlations among carbohydrate metabolism parameters and androgen, adipocytokine, or hs-CRP levels emerged with regard to the PCOS group ( $r$ indicates coefficient of correlation).

\begin{tabular}{llllll}
\hline Parameter 1 & Fasting insulin & G/I & HOMA-IR & ISI & $\Delta$ AUCG \\
Parameter 2 & FAl & FAI & FAI & hs-CRP & FAl \\
$r$ & 0.72 & -0.87 & 0.68 & -0.59 & 0.68 \\
Parameter 1 & AAUCI & $\Delta$ AUCl & 1 st PHIS & Visfatin & \\
Parameter 2 & FAl & hs-CRP & Visfatin & FAI & \\
$r$ & 0.88 & -0.59 & 0.69 & 0.68 & \\
\hline
\end{tabular}


premenopausal PCOS women compared with controls after adjustment for obesity (40), while, in the past, lipocalin-2 levels have been found to be elevated in premenopausal PCOS women (41). In this study, the absence of difference in adipocytokines levels between postmenopausal PCOS and control women is corroborated to the absence of difference in the adipocytokine to waist circumference ratio between the two groups of women. Only lipocalin-2 levels-to-waist circumference ratio was higher in postmenopausal PCOS than in control women, stressing the role of visceral adipose tissue. Of note, in this study, visfatin levels correlated positively with FAI in postmenopausal PCOS women similar to correlations found in PCOS women of reproductive age $(42)$.

In conclusion, early postmenopausal overweight women with PCOS are characterized by hyperinsulinemia, abdominal adiposity, and hyperandrogenism with no difference in insulin resistance compared with control women, indicating that some of the major pathophysiological features of PCOS are present after menopause albeit to a lesser extent. In addition, in postmenopausal PCOS women, androgens correlated positively with OGTT insulin response while insulin secretion was best predicted by FAI levels. Parallel to the amelioration of insulin resistance in PCOS after menopause, adipocytokine levels do not differ between postmenopausal PCOS and non-PCOS women. Further studies in late menopause in PCOS women are needed to clarify the pathophysiological features and the evolution of the correlation between insulin resistance and hyperandrogenism. In face of the persistence of the metabolic and hormonal aberrations in postmenopausal PCOS women, clinical attention is mandatory in order to identify and prevent the disturbances in carbohydrate metabolism and its sequelae.

\section{Declaration of interest}

The authors declare that there is no conflict of interest that could be perceived as prejudicing the impartiality of the research reported.

\section{Funding}

This research did not receive any specific grant from any funding agency in the public, commercial, or not-for-profit sector.

\section{References}

1 Diamanti-Kandarakis E, Kouli CR, Bergiele AT, Filandra FA, Tsianateli TC, Spina GG, Zapanti ED \& Bartzis MI. A survey of the polycystic ovary syndrome in the Greek island of Lesbos: hormonal and metabolic profile. Journal of Clinical Endocrinology and Metabolism $1999 \mathbf{8 4}$ 4006-4011. (doi:10.1210/jc.84.11. 4006)

2 Legro RS, Castracane VD \& Kauffman RP. Detecting insulin resistance in polycystic ovary syndrome: purposes and pitfalls. Obstetrical \& Gynecological Survey 200459 141-154. (doi:10.1097/ 01.OGX.0000109523.25076.E2)
3 Legro RS, Kunselman AR, Dodson WC \& Dunaif A. Prevalence and predictors of risk for type 2 diabetes mellitus and impaired glucose tolerance in polycystic ovary syndrome: a prospective, controlled study in 254 affected women. Journal of Clinical Endocrinology and Metabolism 199984 165-169. (doi:10.1210/jc.84.1.165)

4 Rincon J, Holmang A, Wahlstrom EO, Lonnroth P, Bjorntorp P, Zierath JR \& Wallberg-Henriksson H. Mechanisms behind insulin resistance in rat skeletal muscle after oophorectomy and additional testosterone treatment. Diabetes 199645 615-621. (doi:10. 2337/diabetes.45.5.615)

5 Corbould A. Chronic testosterone treatment induces selective insulin resistance in subcutaneous adipocytes of women. Journal of Endocrinology 2007192 585-594. (doi:10.1677/joe.1.07070)

$6 \mathrm{Qu} \mathrm{J}$, Wang Y, Wu X, Gao L, Hou L \& Erkkola R. Insulin resistance directly contributes to androgenic potential within ovarian theca cells. Fertility and Sterility 200991 1990-1997. (doi:10.1016/ j.fertnstert.2008.02.167)

7 Barber TM, Wass JA, McCarthy MI \& Franks S. Metabolic characteristics of women with polycystic ovaries and oligoamenorrhoea but normal androgen levels: implications for the management of polycystic ovary syndrome. Clinical Endocrinology 200766 513-517. (doi:10.1111/j.1365-2265.2007.02764.x)

8 Azziz R, Woods KS, Reyna R, Key TJ, Knochenhauer ES \& Yildiz BO. The prevalence and features of the polycystic ovary syndrome in an unselected population. Journal of Clinical Endocrinology and Metabolism 200489 2745-2749. (doi:10.1210/jc.2003032046)

9 Cohen B, Novick D \& Rubinstein M. Modulation of insulin activities by leptin. Science $1996 \mathbf{2 7 4} 1185-1188$. (doi:10.1126/science. 274.5290.1185)

10 Fukuhara A, Matsuda M, Nishizawa M, Segawa K, Tanaka M, Kishimoto K, Matsuki Y, Murakami M, Ichisaka T, Murakami H et al. Visfatin: a protein secreted by visceral fat that mimics the effects of insulin. Science $2005 \mathbf{3 0 7} 426-430$. (doi:10.1126/ science.1097243)

11 Esteve E, Ricart W \& Fernandez-Real JM. Adipocytokines and insulin resistance: the possible role of lipocalin-2, retinol binding protein-4, and adiponectin. Diabetes Care 200932 (Suppl 2) S362-S367. (doi:10.2337/dc09-S340)

12 Festa A, D’Agostino R Jr, Howard G, Mykkanen L, Tracy RP \& Haffner SM. Chronic subclinical inflammation as part of the insulin resistance syndrome: the Insulin Resistance Atherosclerosis Study (IRAS). Circulation 2000102 42-47. (doi:10.1161/01. CIR.102.1.42)

13 Tarkun I, Arslan BC, Canturk Z, Turemen E, Sahin T \& Duman C. Endothelial dysfunction in young women with polycystic ovary syndrome: relationship with insulin resistance and low-grade chronic inflammation. Journal of Clinical Endocrinology and Metabolism 200489 5592-5596. (doi:10.1210/jc.2004-0751)

14 Pradhan AD, Manson JE, Rifai N, Buring JE \& Ridker PM. C-reactive protein, interleukin 6 , and risk of developing type 2 diabetes mellitus. Journal of the American Medical Association 2001 286 327-334. (doi:10.1001/jama.286.3.327)

15 Hudecova M, Holte J, Olovsson M, Larsson A, Berne C \& Poromaa IS. Diabetes and impaired glucose tolerance in patients with polycystic ovary syndrome - a long term follow-up. Human Reproduction 201126 1462-1468. (doi:10.1093/humrep/ der065)

16 Winters SJ, Talbott E, Guzick DS, Zborowski J \& McHugh KP. Serum testosterone levels decrease in middle age in women with the polycystic ovary syndrome. Fertility and Sterility $2000 \mathbf{7 3}$ 724-729. (doi:10.1016/S0015-0282(99)00641-X)

17 Markopoulos MC, Rizos D, Valsamakis G, Deligeoroglou E, Grigoriou O, Chrousos GP, Creatsas G \& Mastorakos G. Hyperandrogenism in women with polycystic ovary syndrome persists after menopause. Journal of Clinical Endocrinology and Metabolism 2011 96 623-631. (doi:10.1210/jc.2010-0130)

18 Zawadski JK \& Dunaif A. Diagnostic criteria for polycystic ovary syndrome: towards a rational approach. In Polycystic Ovary Syndrome, pp 377-384. Eds A Dunaif, JR Givens \& F Haseltine. Boston: Blackwell Scientific, 1992. 
19 Durnin JV \& Womersley J. Body fat assessed from total body density and its estimation from skinfold thickness: measurements on 481 men and women aged from 16 to 72 years. British Journal of Nutrition 197432 77-97. (doi:10.1079/BJN19740060)

20 Wilke TJ \& Utley DJ. Total testosterone, free-androgen index, calculated free testosterone, and free testosterone by analog RIA compared in hirsute women and in otherwise-normal women with altered binding of sex-hormone-binding globulin. Clinical Chemistry 198733 1372-1375.

21 Nelson RE, Grebe SK, O’Kane DJ \& Singh RJ. Liquid chromatography-tandem mass spectrometry assay for simultaneous measurement of estradiol and estrone in human plasma. Clinical Chemistry 200450 373-384. (doi:10.1373/clinchem.2003. 025478)

22 Legro RS, Finegood D \& Dunaif A. A fasting glucose to insulin ratio is a useful measure of insulin sensitivity in women with polycystic ovary syndrome. Journal of Clinical Endocrinology and Metabolism 199883 2694-2698. (doi:10.1210/jc.83.8.2694)

23 Katz A, Nambi SS, Mather K, Baron AD, Follmann DA, Sullivan G \& Quon MJ. Quantitative insulin sensitivity check index: a simple, accurate method for assessing insulin sensitivity in humans. Journal of Clinical Endocrinology and Metabolism 2000 85 2402-2410. (doi:10.1210/jc.85.7.2402)

24 Haffner SM, Kennedy E, Gonzalez C, Stern MP \& Miettinen H. A prospective analysis of the HOMA model. The Mexico City Diabetes Study. Diabetes Care 199619 1138-1141. (doi:10.2337/ diacare.19.10.1138)

25 Diamanti-Kandarakis E, Kouli C, Alexandraki K \& Spina G. Failure of mathematical indices to accurately assess insulin resistance in lean, overweight, or obese women with polycystic ovary syndrome. Journal of Clinical Endocrinology and Metabolism 2004 89 1273-1276. (doi:10.1210/jc.2003-031205)

26 Stumvoll M, Mitrakou A, Pimenta W, Jenssen T, Yki-Jarvinen H, Van Haeften T, Renn W \& Gerich J. Use of the oral glucose tolerance test to assess insulin release and insulin sensitivity. Diabetes Care 200023 295-301. (doi:10.2337/diacare.23.3.295)

27 Puurunen J, Piltonen T, Morin-Papunen L, Perheentupa A, Jarvela I, Ruokonen A \& Tapanainen JS. Unfavorable hormonal, metabolic, and inflammatory alterations persist after menopause in women with PCOS. Journal of Clinical Endocrinology and Metabolism 201196 1827-1834. (doi:10.1210/jc.2011-0039)

28 Dunaif A, Segal KR, Futterweit W \& Dobrjansky A. Profound peripheral insulin resistance, independent of obesity, in polycystic ovary syndrome. Diabetes 198938 1165-1174. (doi:10.2337/ diabetes.38.9.1165)

29 Corbould A. Effects of androgens on insulin action in women: is androgen excess a component of female metabolic syndrome? Diabetes/Metabolism Research and Reviews 200824 520-532. (doi:10.1002/dmrr.872)

30 Lindheim SR, Buchanan TA, Duffy DM, Vijod MA, Kojima T, Stanczyk FZ \& Lobo RA. Comparison of estimates of insulin sensitivity in pre- and postmenopausal women using the insulin tolerance test and the frequently sampled intravenous glucose tolerance test. Journal of the Society for Gynecologic Investigation 19941 150-154.

31 Proudler AJ, Felton CV \& Stevenson JC. Ageing and the response of plasma insulin, glucose and C-peptide concentrations to intravenous glucose in postmenopausal women. Clinical Science $1992 \mathbf{8 3}$ 489-494.
32 Escobar-Morreale HF, Luque-Ramirez M \& Gonzalez F. Circulating inflammatory markers in polycystic ovary syndrome: a systematic review and metaanalysis. Fertility and Sterility 201195 1048-1058.e2. (doi:10.1016/j.fertnstert.2010.11.036)

33 Verit FF. High sensitive serum C-reactive protein and its relationship with other cardiovascular risk factors in normoinsulinemic polycystic ovary patients without metabolic syndrome. Archives of Gynecology and Obstetrics $2010 \mathbf{2 8 1} 1009-1014$. (doi:10.1007/s00404-009-1226-6)

34 Kassi E, Dalamaga M, Hroussalas G, Kazanis K, Merantzi G, Zachari A, Giamarellos-Bourboulis EJ \& Dionyssiou-Asteriou A. Adipocyte factors, high-sensitive C-reactive protein levels and lipoxidative stress products in overweight postmenopausal women with normal and impaired OGTT. Maturitas 201067 72-77. (doi:10.1016/j.maturitas.2010.05.004)

35 Horejsi R, Moller R, Rackl S, Giuliani A, Freytag U, Crailsheim K, Sudi K \& Tafeit E. Android subcutaneous adipose tissue topography in lean and obese women suffering from PCOS: comparison with type 2 diabetic women. American Journal of Physical Anthropology 2004124 275-281. (doi:10.1002/ajpa. 10364)

36 Tchernof A \& Poehlman ET. Effects of the menopause transition on body fatness and body fat distribution. Obesity Research 1998 6 246-254.

37 Fasshauer M \& Paschke R. Regulation of adipocytokines and insulin resistance. Diabetologia $2003 \mathbf{4 6}$ 1594-1603. (doi:10.1007/s00125-003-1228-z)

38 Wajchenberg BL. Subcutaneous and visceral adipose tissue: their relation to the metabolic syndrome. Endocrine Reviews 200021 697-738. (doi:10.1210/er.21.6.697)

39 Glintborg D, Andersen M, Hagen C, Frystyk J, Hulstrom V, Flyvbjerg A \& Hermann AP. Evaluation of metabolic risk markers in polycystic ovary syndrome (PCOS). Adiponectin, ghrelin, leptin and body composition in hirsute PCOS patients and controls. European Journal of Endocrinology 2006155 337-345. (doi:10.1530/eje.1.02207)

40 Tan BK, Chen J, Digby JE, Keay SD, Kennedy CR \& Randeva HS. Increased visfatin messenger ribonucleic acid and protein levels in adipose tissue and adipocytes in women with polycystic ovary syndrome: parallel increase in plasma visfatin. Journal of Clinical Endocrinology and Metabolism $2006915022-5028$. (doi:10.1210/jc.2006-0936)

41 Cakal E, Ozkaya M, Engin-Ustun Y \& Ustun Y. Serum lipocalin-2 as an insulin resistance marker in patients with polycystic ovary syndrome. Journal of Endocrinological Investigation 201134 97-100. (doi:10.3275/7077)

42 Kowalska I, Straczkowski M, Nikolajuk A, Adamska A, Karczewska-Kupczewska M, Otziomek E, Wolczynski S \& Gorska M. Serum visfatin in relation to insulin resistance and markers of hyperandrogenism in lean and obese women with polycystic ovary syndrome. Human Reproduction 200722 1824-1829. (doi:10.1093/humrep/dem118)

Received 24 June 2012

Revised version received 29 September 2012

Accepted 17 October 2012 